A TECHNIQUE OF FINGERPRINT RECOGNITION USING

\title{
ARTIFICIAL NEURAL NETWORK
}

\section{SONI $^{1}$ \& SAURABH VERMA ${ }^{2}$}

${ }^{1}$ Research Scholar, Department of Electronics and Communication Engineering, Moradabad Institute of Technology, Moradabad, Uttar Pradesh, India

${ }^{2}$ Assistant Professor, Department of Electronics and Communication Engineering, Moradabad Institute of Technology,

Moradabad, Uttar Pradesh, India

ABSTRACT
This paper is present fingerprint recognition with the technique of artificial neural network. Fingerprint is
widely accepted for personnel identification. In this technique we used back propagation algorithm. Firstly we used filter
and histogram process of the input image than we trained artificial neural network on the input image.
KEYWORDS: Fingerprint, Back Propagation Algorithm, Recognition \& Artificial Neural Network

Received: Oct 05, 2017; Accepted: Oct 25, 2017; Published: Jan 06, 2018; Paper Id.: IJECIERDFEB20181

\section{INTRODUCTION}

A fingerprint recognition technique is a biometric system. Artificial neural network made much better a recognition system because it provide high level accuracy. So ANN is used many ways of recognition system. ANN is widely used in biometric system. In this paper we give a very easy technique of recognition with the help of ANN.

\section{LITERATURE SURVEY}

Anil K. Jain and et al [1] described in Pores and ridges: He gives a theory of artificial neural network of three level theories like level one, level two and level three. Level 3 features to assist in identification, automated fingerprint identification systems (AFIS). Without these levels recognition is not possible.

Haykin S. [3] developed in Neural Networks: he gives a technique that recognition a sound and word. It has two types: discrete word and continuous speech recognition systems. Any type can be sub-divided into two categories as Speaker Dependent and Speaker Independent recognition systems.

A. Askarunisa and et al [6] proposed in Finger Print Authentication Using Neural Networks a multiple circular path convolution neural network system for detection of fingerprint. Multiple circular path convolution neural network architecture specifically designed for the analysis of images and fingerprint like structure has been constructed. Author first divided each suspected fingerprint area into sectors and computed the defined mass features for each sector independently.

\section{Overview of Artificial Neural Network}

Figure 1 shows the architecture of artificial neural network. In this we give overview of neurons. This 
figure configuration of neurons. It has three layers input layer, hidden layer and output layer. We give many inputs than we get one output trained from artificial neural network. Output layer have functions used output neurons.

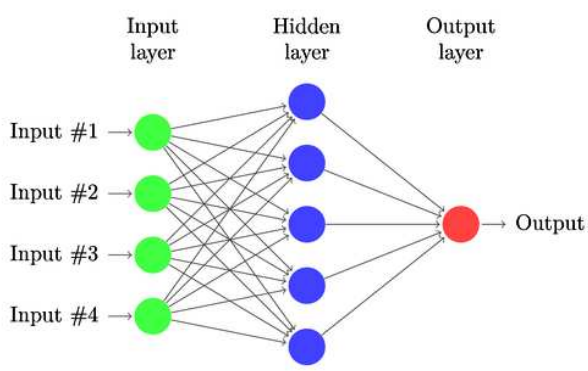

\section{Figure 1: Architecture of Artificial Neural Network with Input, Hidden and Output Layer ANN Defined as a Group of Interconnected Neurons used for Information Processing}

\section{Texture Features}

\section{Circularity}

Circularity of the shape of the region is defined by the value of compactness, $\mathrm{M}$, as defined using the following equation.

$$
\mathrm{C}=4 \pi \text { Area/Perimeter }{ }^{2}
$$

\section{Skewness}

Skewness is defined by S. Skewness is given in the below equation:

$$
\mathrm{S}=\frac{1}{M N} \sum_{i=1}^{M} \sum_{j=1}^{N}\left(\frac{p[(i, j)-\mu]}{\sigma}\right)^{3}
$$

\section{Energy}

Energy is defined by E. Energy is given below equation.

$$
\mathrm{E}=\sum_{i, j} p^{2}(i, j)
$$

\section{Contrast}

The Contrast is expected to be low if the gray levels of the pixels are similar. Contrast has given equation.

$$
\mathrm{C}=\sum_{i} \sum_{j}(i-j)^{2} \mathrm{p}(i, j)
$$

\section{Variance}

The dispersion of the values around the mean is represented by variance. It has given equation.

$$
V=\sum_{i, j=1}^{N}(i-j)^{2} p(i, j)
$$

\section{Perimeter}

The perimeter $(\mathrm{P})$ is equal to the sum of side the side lengths.

$\mathrm{P}=\sum$ side lengths 


\section{METHODOLOGY}

As per the mentioned objectives, to implement a method for evaluating performance of fingerprint recognition images the following steps are to be performed:

- Firstly to obtain the data from database.

- Apply the image enhancement technique such as histogram equalization on input images.

- Then, segment the image.

- Next, extracting texture features.

- Next, feed the features to feed artificial neural network.

- Finally, we get artificial neural network trained result.

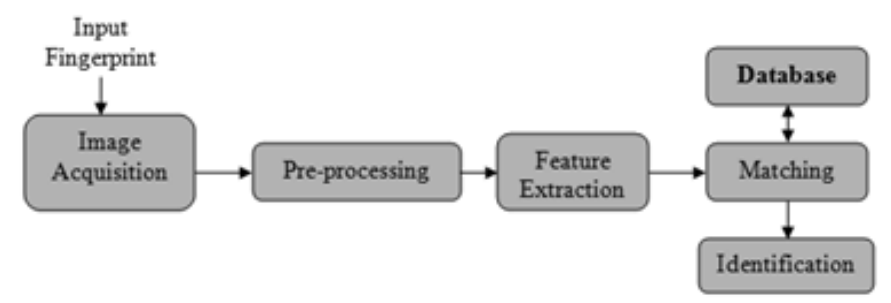

Figure 2: Fundamental Steps of Fingerprint Recognition System

\section{Image Acquisition}

Our system acquires fingerprints in two ways. One is selecting an image file from a collection of fingerprint images from a number of publicly available databases. The second way is to directly capture fingerprint using Biometrical His can-PRO 1000 PPI scanner that interacts with our software. Sensor is capable of dual resolution scanning in both 500 PPI and 1000 PPI. This sensor is a FBI-certified product so it can be used for forensic applications as well.

\section{Fingerprint Image Processing}

Fingerprint image processing operations are image enhancement, image normalization and image binarazation.

\section{Feature Matching}

After the classification of all image blocks in the original fingerprint, a final feature vector is produced. In this feature vector, each extracted feature is represented by its $\mathrm{x}$ and $\mathrm{y}$ coordinate, orientation expressed in degrees and shape type. The database of fingerprints, represented by their feature vectors, is searched and matched against the unknown fingerprint. Feature vector from the database with highest similarity score and exceeding the decision threshold is selected as the resulting identity of the unknown fingerprint.

\section{Fingerprint Classifier}

Classification is the final stage of any image-processing system where each unknown pattern is assigned to a category. The degree of difficulty of the classification problem depends on the variability in feature values for objects in the same category, relative to the difference between feature values for objects in different categories. In this study MLP (Multilayer Perception) classifiers used as pattern classifiers. 


\section{Simulated Result}
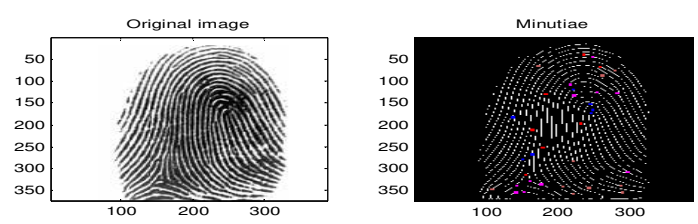

Figure 3: Original Image and Minutiae

These minutiae points' ridge ending and ridge bifurcations are mostly used for verification. Figure 3 gives an idea about terminations and bifurcations. It is very difficult to increase the accuracy of the system in a relevant way by using only one form of representation or only one matching algorithm after the classification of all image blocks in the original fingerprint, a final feature vector is produced. In this feature vector, each extracted feature is represented by its $\mathrm{x}$ and $\mathrm{y}$ coordinate, orientation expressed in degrees and shape type.

Figure 4 Process of passing pixels from the original fingerprint to input layer of neural network for classification algorithms. It is very fast and often outperforms its competitors in recognition rate. This algorithm compares two fingerprint representations and produces a similarity score which varies in the range FMR-FNMR (where FNMR stands for no similarity and FMR is the highest possible similarity measured in our tests). The database of fingerprints, represented by their feature vectors, is searched and matched against the unknown fingerprint. Feature vector from the database with highest similarity score and exceeding the decision threshold is selected as the resulting identity of the unknown fingerprint.

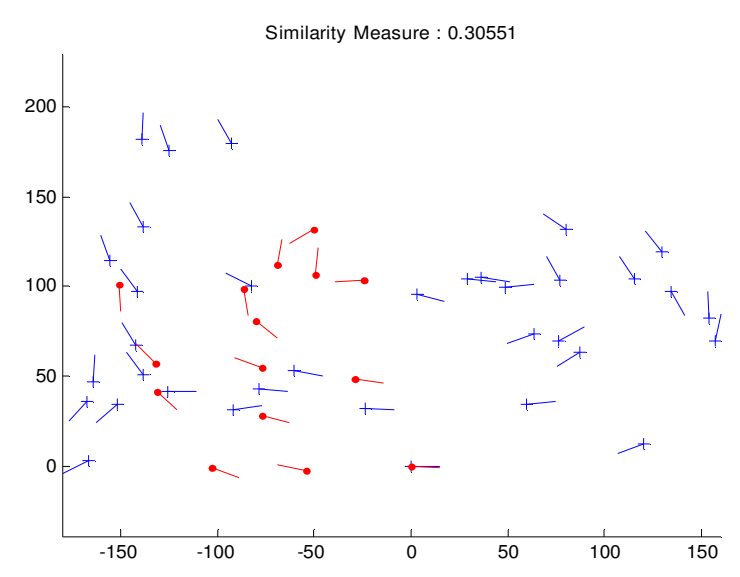

Figure 4: Features Matching Result

\section{RESULTS}

Artificial neural network is broadly used for training large data set because it provides good results in complicated conditions. Neural networks have many advantages over other methods like adaptive learning, self organization, real- time operation, fault tolerance. It has several applications such as hand writing and type writing recognition, fraud detection, criminal sentencing, optimization, pattern recognition etc. 


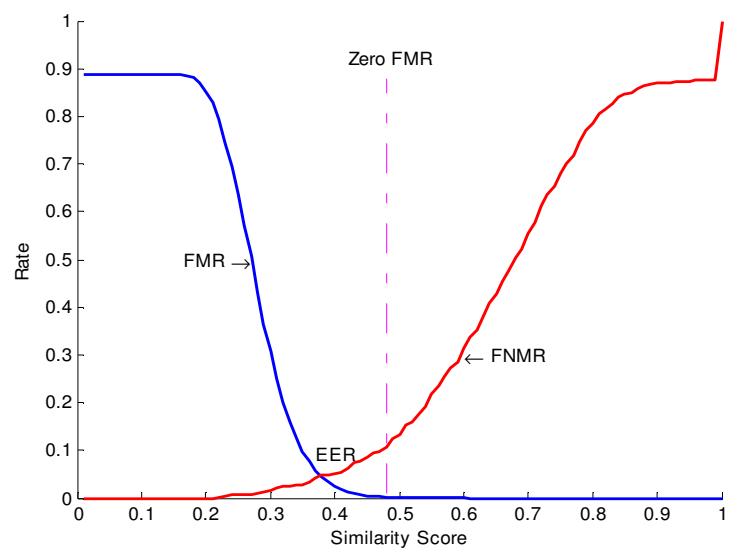

Figure 5: Matching and Non Matching Fingerprint Graph

Figure 5 shows the result of fingerprint matching and non matching features. In this graph we see that matching result is greater than of non matching features result. Here, trainlm function is applied for training network so that it can produce different result sets. The response of the Neural Network is dependent upon weights, biases and activation functions. The activation functions used in the feature extraction neural network are Tangent sigmoid (tensing) used in hidden layer and prelim used in output layer.

These functions acts as summation junction and calculates the output from the inputs presented. After the training phase is completed, the identification process must be implemented in order to evaluate the proposed system. The evaluation process is accomplished by testing the system with known and newly fingerprints images. New images for testing are applied to the trained neural network along with already trained images for calculating the percentage of accuracy and error.

\section{CONCLUSIONS}

A method of fingerprint recognition based on Artificial Neural Network is presented here. Firstly de-noise the images and apply histogram equalization technique for enhancement images. Than extract the features and trained the neural network. Experimental results show that the presented method has the better recognition compared with the previous logic based recognition methods. Moreover, proposed this method is simple and it takes less time for iterations. Therefore, it is effective in terms of time consuming and precision.

\section{REFERENCES}

1. Anil K. Jain, Yi chen, Melten Demirkus, "Pores and ridges: High resolution fingerprint matching using Level 3 features, "IEEE Transactions on Pattern Analysis and Machine Intelligence, vol. 29, no.1, pp. 1-13, Jan 2007.

2. X. Xia, and L. O'Gorman, "Innovations in Fingerprint Capture Devices”, Journal of Pattern Recognition, Pergamum Press, Vol. 36, No. 2, pp. 361-370, 2002.

3. Haykin, S., Neural Networks: “A Comprehensive Foundation”, 2nd ed. (Englewood Cliffs, NJ: Prentice-Hall), 1999.

4. Vijaya Sathiaraj, “A Study on the Neural Network Model for Finger Print Recognition” International Journal Of Computational Engineering Research Vol. 2 Issue. 5, Oct 2012.

5. G. Jha "Artificial Neural Networks", Indian Agricultural Research Institute, PUSA, New Delhi-110 012, 2005. 
6. Askarunisa, Sankaranarayanan. K. Sundaram $R$ and Sathick M. Batcha, "Finger Print Authentication Using Neural Networks" MASAUM Journal of Computing Vo. 1, No. 2, 2009.

7. P. Baldi and Y. Chauvin, "Neural Networks for Fingerprint Recognition", neural computation, 5, pp.485-501, 1993.

8. R. Patil (Waghjale), S. R. Suralkar, "Fingerprint Classification using Artificial Neural Network”, International Journal of Emerging Technology and Advanced Engineering (ISSN 2250-2459, Volume 2, Issue 10, September 2012.

9. D. Maltoni, D. Maio, A. K. Jain and S. Prabhakar, Handbook of Fingerprint Recognition, Springer Professional Computing, Berlin, 2nd ed. 2009.

10. Arun Ross, Anil Jain, James Reisman, “A hybrid fingerprint matcher,” Pattern Recognition, vol. 36, pp. 1661-1673, 2003. 